\title{
Desafiando medos: relatos de enfrentamento de usuários com transtornos fóbico-ansiosos
}

\author{
Challenging fears: reports of users coping with phobic-anxious disorders \\ Desafiando temores: relatos de usuarios frente a trastornos fóbico-ansiosos
}
Priscylla Araújo Almeida', Priscilla Maria de Castro Silva", Lawrencita Limeira Espínola", Elisângela Braga de Azevedo", Maria de Oliveira Ferreira Filha"I
'Centro de Ensino Superior e Desenvolvimento, Faculdade de Ciências Médicas de Campina Grande, Curso de Graduação em Enfermagem (Graduanda). Campina Grande-PB, Brasil.
"Universidade Federal da Paraíba, Centro de Ciências da Saúde, Programa de Pós-Graduação em Enfermagem (Pós-graduandas). João Pessoa-PB, Brasil.
III Universidade Federal da Paraíba, Centro de Ciências da Saúde,
Programa de Pós-Graduação em Enfermagem. João Pessoa-PB, Brasil.

\begin{abstract}
Submissão: 29-07-2011 Aprovação: 15-06-2013
RESUMO

Estudo interpretativo e compreensivo que objetivou investigar as formas de enfrentamento utilizadas pelos portadores de transtornos fóbico-ansiosos do Centro de Atenção Psicossocial I, do município de Queimadas-PB, Brasil, no período de outubro a dezembro de 2010. O material empírico foi produzido por meio de entrevistas e submetido à análise de conteúdo proposta por Bardin. Desse material, foram extraídos os temas principais que formaram a categoria temática denominada CAPS I como lugar de expressão da subjetividade, e os eixos temáticos Reconhecimento da doença; Solidão e isolamento; e Família como principal ponto de apoio. Os resultados identificaram que, ao aderir ao tratamento, os usuários começaram a resgatar a autoestima, fortalecer vínculos e a enfrentar os sintomas biopsicossociais. Ressalta-se a relevância da dimensão afetiva no encontro entre profissionais, usuários e familiares. Conclui-se que a prática humanizada garante e estimula a integração entre os processos de trabalho e os projetos de vida dos usuários. Descritores: Serviços de Saúde Mental; Sofrimento Psíquico; Relações Familiares.
\end{abstract}

\section{ABSTRACT}

This is an interpretative and comprehensive study that aimed to investigate ways of coping used by patients with phobic-anxious disorders, attended at the Centro de Atenção Psicossocial I, of the municipality of Queimadas, state of Paraíba, Brazil, in the period from October to December 2010. The empirical data were obtained through interviews and subjected to content analysis. From this material were extracted the main themes that formed the thematic category called CAPS I as a space to expression of subjectivity, and the thematic axis: Recognition of the illness; Loneliness and isolation; and Family as the primary point of support. The results showed that, while adhering to treatment, users began to recover their self-esteem, strengthen bonds and to deal with the bio psychosocial symptoms. We emphasize the importance of the affective dimension in the encounter between professionals, patients and families. It can be concluded that the humanized practice ensures and stimulates the integration between the work processes and the users' life projects. Key words: Mental Health Services; Psychological Distress; Family Relationships.

\section{RESUMEN}

Estudio descriptivo-exploratorio que objetivó investigar las formas utilizadas por portadores para enfrentamiento de trastornos fóbico-ansiosos del Centro de Atención Psicosocial I, de la Comunidad de Queimadas, estado de Paraíba, Brasil, en el período de octubre a diciembre del 2010. El material empírico fue producido por medio de entrevistas y sometido al análisis del discurso propuesta por Bardin. De este material, fueron extraídos los temas principales que formarán la categoría temática CAPS I como lugar de expresión de subjetividad, y de los ejes temáticos: Reconocimiento de la enfermedad; Soledad y aislamiento; Familia como principal punto de apoyo. Los resultados identificarán que, al adherir al tratamiento, los usuarios empezarán a rescatar su autoestima, fortalecer vínculos y a enfrentar los síntomas biopsicosociales. Se resalta la relevancia de la dimensión afectiva en el encuentro entre profesionales, usuarios y familiares. Se concluye que la práctica humanizada garantiza y estimula la integración entre los procesos de trabajo y los proyectos de vida de los usuarios.

Palabras clave: Salud Mental; Trastorno Mental; Relación Familiar.

\section{AUTOR CORRESPONDENTE Lawrencita Limeira Espinola}

E-mail: lawrencita_@hotmail.com apresentado ao Curso de Graduação em Enfermagem, Faculdade de Ciências Médicas. Campina Grande-PB, Brasil. 


\section{INTRODUÇÃO}

Atualmente, segundo estimativas da Organização Mundial da Saúde (OMS), mais de 450 milhões de pessoas no mundo sofrem com algum tipo de transtorno mental. No Brasil, 23 milhões de pessoas necessitam de algum atendimento em saúde mental e pelo menos 5 milhões de brasileiros sofrem com transtornos mentais graves e persistentes. Nesse universo, encontram-se doenças, como depressão, suicídio, transtornos de ansiedade, dependência de álcool e drogas ${ }^{(1)}$.

No Brasil, em torno de $31 \%$ a $50 \%$ da população tende a apresentar, durante a vida, pelo menos um episódio de algum transtorno mental. Por causa desses transtornos, cerca de $20 \%$ a $40 \%$ da população necessita de ajuda profissional, indicando a relevância social da problemática. Muitas dessas pessoas sofrem em silêncio, sozinhas, e não recebem tratamento adequado. Entre o sofrimento e as perspectivas de tratamento existem barreiras de estigma, preconceitos e exclusão. Esse cenário vem forçando os governos de vários países a desenvolver políticas públicas que priorizem a prevenção e o tratamento da saúde mental, colocando a temática como uma necessidade urgente ${ }^{(1)}$.

Para enfrentar tal malefício, é necessária uma série de medidas, como diagnóstico precoce e acompanhamento por uma equipe especializada, proporcionando ao portador de transtorno mental uma oportunidade de atendimento biopsicossocial, terapia medicamentosa, terapia comunitária, terapia familiar e individual, abandono do grupo de pares usuários, mudança de atitudes e hábitos, visando à reinserção social.

Apesar da existência de ampla rede de atenção, documentos oficiais da última década ratificam a ênfase do provimento do cuidado e atenção à saúde de portadores de necessidades especiais, por ser este uma estratégia fundamental para o acesso e acolhimento de pacientes e suas famílias aos serviços de saúde e por considerar a resolutividade nesse nível de atenção à saúde ${ }^{(2)}$.

Um dos fatores que tem influenciado esses avanços foi a implantação dos Centros de Atenção Psicossocial (CAPS), criados oficialmente a partir da Portaria GM 224/92 e regulamentados pela Portaria n 336/GM, de 19 de fevereiro de 2002, integrando, desse modo, a rede do Sistema Único de Saúde (SUS), e que gradativamente, tem assumido a posição de coordenador da assistência aos usuários portadores de sofrimentos psíquico, tendo em vista as suas características básicas ${ }^{(2)}$.

Trata-se de um serviço de referência para o tratamento de pessoas que sofrem com transtornos mentais, cuja severidade justifique sua permanência num serviço de cuidado intensivo, que proporciona um acompanhamento clínico e a reinserção social dos usuários pelo acesso ao trabalho, lazer, exercício dos direitos civis e fortalecimento dos laços familiares e comunitá$\operatorname{rios}^{(3)}$. Além disso, realizam-se diversas ações no sentido de enfrentar a exclusão das pessoas portadoras de transtornos psicossociais. Esse foi um marco nesse processo de criação de uma rede intersetorial de enfrentamento, mapeamento das situações de sofrimento psíquico, acolhimento e assistência à vítima.

Destacam-se o atendimento individual, em grupo, em oficinas terapêuticas bem como o atendimento à família, visitas e atendimentos domiciliares e atividades comunitárias, enfocando a integração social do usuário na família e na comunidade. Algumas atividades são específicas de determinados profissionais, porém as mais importantes são aquelas realizadas individualmente ou em grupo, que implicam escuta, acolhimento, estímulo para a vida, para a autonomia, para a cidadania ${ }^{(4)}$.

A equipe técnica do CAPS deve valorizar a atuação interdisciplinar, permitindo, com isso, um enfoque ampliado dos problemas de saúde, identificados tanto nos serviços quanto nos usuários cadastrados e acompanhados no serviço. Ressalta-se que os vínculos terapêuticos estabelecidos entre ambos, podem ser parcialmente mantidos em esquema flexível, o que pode facilitar a trajetória com mais segurança em direção à comunidade, ao seu território reconstruído e ressignificado ${ }^{(2)}$.

No entanto, o papel da enfermagem é importante, tendo em vista a valorização na manutenção de um ambiente que favoreça a liberdade, o acolhimento, o respeito às pessoas e às leis da população, de estímulo às habilidades e às capacidades emergentes ${ }^{(5)}$.

Deve-se ter em mente que, no tratamento desses usuários, mesmo quando não é possível trabalhar com a hipótese de remissão total do problema, a obtenção de progressos no nível de desenvolvimento, em qualquer aspecto de sua vida mental, pode significar melhora importante nas condições de vida para eles e suas famílias ${ }^{(2)}$.

Salienta-se que os transtornos de ansiedade estão entre as condições psiquiátricas mais prevalentes nos Estados Unidos. Além disso, observa-se que eles produzem morbidade desordenada, uso exagerado de serviços de saúde e comprometimento do desempenho. Os pacientes com transtornos de ansiedade tendem a reagir de maneira excessiva ao perigo e subestimam sua capacidade de lidar com as ameaças percebidas ao seu bem-estar físico e psicológico ${ }^{(6)}$.

A ansiedade e o medo passam a ser reconhecidos como patológicos quando são exagerados, desproporcionais em relação ao estímulo, ou qualitativamente variados, e interferem na qualidade de vida, no conforto emocional ou no desempenho diário da pessoa(7).

A neurose fóbica é caracterizada pela sistematização da angústia sobre pessoas, coisas, situações ou atos que se tornam objeto de terror paralisante. Corresponde a estado de perturbação da saúde mental, no qual a fobia é o sintoma prevalente; como resultado do mecanismo de defesa, encontra-se a angústia ${ }^{(8)}$. Em outras palavras, a angústia é um sentimento indiferenciado, e a fobia é esse sentimento que se particulariza em determinadas coisas, situações, pessoas.

Os níveis intensos de ansiedade social podem provocar um impacto negativo sobre o desenvolvimento e bem-estar dos jovens. Diante disso, os quadros patológicos irão manifestar-se através do grau de dificuldade para lidar com os medos, do prejuízo sobre o funcionamento social ou acadêmico-ocupacional e da gravidade do desconforto subjetivo sentido. Variadas reações excessivas, como ataques de pânico e de choro, queixas somáticas excessivas, acessos de raiva, e comportamento que evitem grande parte de situações e atividades, sugerem um quadro clínico de fobia social ${ }^{(9)}$.

As fobias manifestam-se em tipos específicos, que comportam 
divisões: fobias simples, fobias sociais, síndrome de pânico. A maioria das fobias sociais inicia-se na puberdade, sem predominância entre os sexos. Ordenadamente há tendência de se reduzir todas as neuroses fóbicas ou fobias à síndrome do pânico, ou ainda reuni-las sob a rubrica transtorno de ansiedade ou transtorno fóbico-ansioso ${ }^{(8)}$.

Assim, partindo da premissa de que o trabalho em saúde mental é ainda um desafio, entende-se que é importante analisar as formas de enfrentamento utilizadas por pessoas portadoras de transtornos fóbicos que são atendidas no CAPS. Salienta-se que esse serviço é responsável pela atenção psicossocial de toda a população brasileira.

Estudos em saúde realizados sob a ótica sociocultural reconhecem que a doença e a saúde se constituem de vários significados: as pessoas constroem esses significados através da sua realidade social. Essa representação de significados é passível de diferentes interpretações, conforme o contexto social no qual a pessoa vive. Por isso, cada vez mais verificamos que, no campo da saúde coletiva, são desenvolvidos estudos sobre os significados e as formas de enfrentamentos utilizadas por pessoas com sofrimento psíquico ${ }^{(10)}$.

Como cada pessoa tem um discurso próprio sobre o corpo, saúde e doença, o qual pode variar entre as classes sociais, pode-se pensar que, em termos de saúde mental, essa diversidade de concepções determina diferentes expectativas, notando-se, muitas vezes, uma defasagem importante entre as necessidades e as prioridades avaliadas por profissionais e pelos usuários dos serviços de saúde. Como decorrência, defasagem parecida pode ocorrer quando se trata de identificar intervenções capazes de atender a essas necessidades ${ }^{(10)}$.

Estas considerações motivaram o presente estudo, cujo objetivo foi identificar quais os usuários do CAPS I, na cidade de Queimadas - PB, que possuem diagnóstico de transtorno fóbico-ansioso e analisar as formas de enfrentamento utilizadas por ele para melhorar os sintomas desse transtorno.

\section{METODOLOGIA}

Trata-se de uma pesquisa compreensiva e interpretativa de abordagem qualitativa, realizada com vinte usuários do Centro de Atenção Psicossocial (CAPS) I, denominado "Integração", no município de Queimadas, estado da Paraíba, Brasil, no período de outubro a dezembro de 2010. Esse serviço foi escolhido para ser objeto de estudo por ser o único que atende aos usuários com transtornos mentais.

A pesquisa privilegiou contexto natural, envolvendo a observação de situações reais e cotidianas. O material empírico foi coletado através de entrevista com um questionário com questões semiestruturadas, que foram analisadas utilizando-se a Análise de Conteúdo de Bardin ${ }^{(11)}$. Esta é uma técnica de investigação que parte de uma descrição objetiva e sistemática do conteúdo das comunicações, tendo uma organização própria no procedimento da análise, que permite a inferência de conhecimentos relativos às condições de produção/recepção dessas mensagens.

Os participantes assinaram o Termo de Consentimento Livre e Esclarecido, sendo-lhes assegurado o anonimato absoluto das informações, assim como a privacidade. As falas dos profissionais foram identificadas pela letra $E$, seguida do número da entrevista como forma de garantir seu anonimato.

O estudo foi aprovado pelo Comitê de Ética em Pesquisa do Centro de Ensino Superior e Desenvolvimento - CESED, Protocolo $\mathrm{n}^{\circ}$ 4814.0.000.405-10, seguindo os ditames da Comissão Nacional de Ética em Pesquisa (CONEP).

$\mathrm{Na}$ análise, os dados foram ordenados a partir da leitura detalhada dos discursos de cada usuário entrevistado. Esses discursos foram transcritos com a finalidade de nos familiarizar com a linguagem dos entrevistados, buscando uma visão geral de cada um. Um arquivo foi criado com todas as entrevistas para ajudar na categorização. O material empírico foi ordenado com a leitura das falas transcritas, enfatizado como um processo hermenêutico em que o material empírico é transformado em um corpus a ser tecnicamente trabalhado ${ }^{(12)}$. Dessa maneira, foi feita uma leitura das falas, horizontal e exaustiva, dando ênfase à riqueza dos discursos.

Para a análise do material empírico, foram transcritas e digitadas as entrevistas, sendo, na sequência, realizadas leituras exaustivas para construção das categorias. Durante a análise, mantivemos atenção às particularidades de cada relato da entrevista, bem como à totalidade deles, buscando através da análise de conteúdo as categorias temáticas. Essas unidades de significação foram extraídas do texto analisado, cuja frequência de aparição demonstrava significado para elucidação do objetivo do estudo ${ }^{(11)}$.

\section{RESULTADO}

No período da realização da pesquisa, estavam cadastrados 1.500 usuários no serviço, com diferentes transtornos mentais que variavam de leve a grave; e os serviços prestados variavam em três modalidades: intensiva, semi-intensiva e não intensiva. Dentre esses usuários, 45 apresentavam transtornos fóbico-ansiosos em seus registros de prontuários, porém apenas 20 aceitaram participar da pesquisa.

Dos entrevistados para o estudo, $86 \%$ eram do gênero feminino e $90 \%$ encontravam-se na faixa etária entre 30 e 55 anos. Em relação ao nível escolar, 80\% dos participantes possuíam o fundamental completo. A maior parte deles - 93\% - residia na zona urbana. Verificou-se que 70\% desses entrevistados estavam em tratamento no CAPS I há três anos, e que 29\% apresentavam o mesmo diagnóstico: CID-F 41.

O resultado das entrevistas possibilitou o surgimento de uma categoria temática denominada: O CAPS como espaço de descoberta de transtornos fóbicos; e, a partir dela, os eixos temáticos: Aceitação e procura de tratamento; $\mathrm{O}$ peso da doença; Os desafios enfrentados no convívio social; Família como principal ponto de apoio; e Mudanças na qualidade de vida dos usuários do CAPS.

\section{ANÁLISE E DISCUSSÃO DO MATERIAL EMPÍRICO}

As práticas realizadas no CAPS pelos profissionais de saúde preocupam-se com o sujeito e sua singularidade, sua história, sua cultura e sua vida quotidiana. Essas ocorrem em ambiente 
acolhedor e, muitas vezes, ultrapassam a própria estrutura física, em busca da rede de suporte social ${ }^{(2)}$.

\section{A. O CAPS como espaço de descoberta de transtornos fóbicos}

Apesar do aumento da demanda de pessoas com problemas em decorrência de transtornos mentais, chama atenção dos profissionais da saúde do CAPS para o número reduzido de portadores de transtornos fóbicos-ansiosos que aderem ao tratamento dessa doença.

Um estudo recente enfatizou a necessidade de inovar a prática diária do profissional da saúde, para que o atendimento não se limite ao cenário do CAPS, e realmente assuma o compromisso com os pressupostos da Reforma Psiquiátrica, que é complexa e envolve aspectos biológicos, sociais, políticos e culturais do indivíduo em sofrimento psíquico ${ }^{(13)}$.

A sociedade necessita de serviços com profissionais da saúde, que realizem práticas que ajudem as pessoas com transtornos mentais e seus familiares a identificar e potencializar os recursos internos e externos para viver socialmente, exercendo sua cidadania, com liberdade, saúde, educação, lazer e todos os direitos civis.

Nesse contexto, os profissionais do CAPS I Integração têm desenvolvido esforços no sentido de acolher o usuário e identificar qual o transtorno que o fez procurar tratamento. E após uma avaliação minuciosa de toda a equipe, estabelece-se um Projeto Individual Terapêutico que irá auxiliar esse usuário a reconhecer que está doente e enfrentar o sofrimento psíquico que interfere na qualidade de vida da pessoa.

Em relação à descoberta da doença e aos mecanismos de aquisição das fobias, há evidências de que alguns medos são adquiridos por condicionamento ou outras formas de aprendizado, enquanto outros surgem de forma espontânea ou não associativa, como se percebe nas falas dos entrevistados ${ }^{(14)}$.

[...] Desde 2006, quando começou com uma tontura e coração acelerado, começou com um estresse. (E01).

[...] Foi depois da separação do meu marido, eu senti uma raiva não tanto por mim, mas por ele. Foi daí que eu passei mal e fui para o hospital. Sempre ia, mas eu não tinha nada. Só disseram que meu problema era nervo. (E04).

[...] Desde que tive meu filho, há 25 anos, tive que tomar medicação [...] porque ele nasceu deficiente, fiquei triste e comecei a ficar ansiosa, engordando muito, sempre com vontade de chorar. (E08).

O relato de ansiedade dos participantes está relacionado a um medo irracional que foi desencadeado conscientemente por um objeto, atividade ou situação específica temida. E o sofrimento fóbico acarreta alteração da capacidade funcional do indivíduo.

De acordo com o DSM-IV-TR, o diagnóstico será confirmado quando há presença de medo excessivo e persistente de situações nas quais a pessoa julga estar exposta à avaliação de outros, ou há comportamento humilhante ou vergonhoso, levando a consequências prejudiciais no ambiente familiar, social ou ocupacional(7).

\section{Aceitação e procura de tratamento}

O percurso entre a aceitação da patologia até a procura de ajuda profissional muitas vezes se torna bastante difícil e demorado para os indivíduos, pois eles enfrentam vários tipos de preconceitos.

É importante salientar que a procura por uma equipe de profissionais capacitados para realizar o tratamento de transtornos mentais é fundamental para que o paciente possa controlar os sintomas apresentados. Assim, é fundamental que a pessoa procure ajuda dos profissionais do CAPS para tentar ter uma melhor qualidade de vida, uma vez que existem vários tratamentos que auxiliam em sua recuperação.

Os indivíduos que apresentam algum tipo de fobia, geralmente, reconhecem que o medo é excessivo ou irracional, mas não o dominam. Assim, evitam pessoas pela situação temida; e, se sabem antecipadamente que têm que deparar com ela, desenvolvem uma ansiedade que piora o desempenho na hora de enfrentarem o fato temido. Dessa forma, há um aumento da ansiedade e, assim, num círculo vicioso, o desempenho vai piorando ${ }^{(8)}$. O medo e a esquiva acabam influindo significativamente na vida do usuário.

[...] Primeiro eu procurei os médico do posto lá de onde eu morava. Demorei, o CAPS foi mais recente para mim, porque já tenho esse problema há 14 anos. (E05).

\section{[...] para procurar o Psiquiatra, demorou muito. (E06).}

[...] Fui atendido por um clinico geral, depois um cardiologista, ai depois o cardiologista pediu para eu procurar um neurologista, ai eu procurei um doutor... em Campina Grande. Ele me examinou e pediu um eletroencefalograma e não deu nada. Pelos sintomas que apresentei, ele disse que era uma ansiedade muito forte. Eu demorei a procurar o CAPS, porque não sabia o que acontecia no CAPS. Depois descobri que uma prima trabalhava aqui nesse CAPS, aí vim para cá. (E15).

De acordo com os relatos, é possível identificar que o diagnóstico dos Transtornos Fóbico-ansiosos muitas vezes são feitos por médicos generalistas e não especialistas, pois se tratam das primeiras procuras dos pacientes. Para descobrir se realmente há a presença do transtorno, a investigação deve ser associada à outra patologia clínica, pois o diagnóstico pode ser mais difícil apenas com os sintomas sem associação de outros. Como exemplo, o transtorno de fobia social junto com depressão, pois este muitas vezes, é diagnosticado como depressivo e não como origem do transtorno ${ }^{(15)}$.

\section{O peso da doença}

Pode-se dizer que o primeiro passo do tratamento é sensibilizar o paciente de que a fobia social é uma doença, e por isso pode e deve ser tratada, o que, geralmente, não é difícil, pois o sofrimento significativo torna esses pacientes receptivos à possibilidade de ajuda ${ }^{(14)}$. 
[...] Eu só faço chorar tem hora que eu choro por besteira e agora é pior que um filho meu faleceu faz um mês e eu tinha um homem que teve AVC e vivia dentro de casa, ai eu tinha que cuidar. (EO2).

[...] Eu fico sem sair, sem ver as pessoas, sem querer conversar e fico muito, muito assim aborrecida e de repente eu começo a amolecer e ficar na cama. Sei não. (E05).

[...] Acontecia desde pequeno, quando eu tinha oito anos de idade, quando eu caí de um jumento e minha mãe pensou que eu tinha sido atropelado, aí ela quebrou o resguardo, aí eu me senti culpado, porque ela ficou doente depois disso, aí eu sempre ficava pensando que era por causa de mim, por causa de mim e eu sempre fui uma pessoa que me preocupo muito com a família, aí eu sentia tremores, endurecimento e suava. (E01).

Esse processo produz um medo excessivo de humilhação ou embaraço em vários contextos sociais, como comer, falar, escrever, praticar atividades físicas em público, assim como também urinar em banheiro público. O resultado disso é uma importante limitação na vida da pessoa devido à não aceitação dessas situações ou atividades sociais temidas; bem como podem ocorrer prejuízos na vida profissional e afetiva do paciente ${ }^{(16)}$.

As fobias apresentam como tratamento a combinação de terapia cognitivo- comportamental, o uso de medicamentos, a psicoterapia, a terapia de dessensibilização e terapia de exposição. Dessa maneira, pode-se dizer que a perspectiva de recuperação é positiva, mas é preciso aprender a controlar essa sensação e permitir que o organismo também consiga ficar livre da reação que sente quando se está em crise $^{(7)}$.

\section{Os desafios enfrentados no convívio social}

Os indivíduos com transtornos de ansiedade sentem dificuldade em conviver socialmente, por apresentar sintomas de medo e vergonha.

\section{[...] Eu prefiro ficar só. (E05).}

[...] De várias formas, entro em pânico, às vezes dá vontade de ficar sozinha. Depois eu digo para mim mesma, que não vou ficar sozinha. Começo a ficar com as pessoas, procuro Deus, procuro a igreja. Fico feliz, bem e percebo que não é nada disso do que eu pensava. (E08).

[...] Tem momentos que eu quero correr, andar e ficar sozinha. (E17).

O enfrentamento dos sintomas do transtorno traz para a vida da pessoa portadora várias modificações, pois esse transtorno proporciona-lhe momentos de exclusão social. De acordo com as análises coletadas, observou-se a frequência com que os entrevistados têm de se isolar, prejudicando assim sua vida, tanto pessoal como profissional. Dessa forma, a principal função da equipe de saúde do CAPS é encontrar ferramentas para diminuir o sofrimento psíquico do paciente e torná-lo apto a conviver socialmente, superando os obstáculos diários e vivendo em paz no meio em que vive.

O portador de transtorno fóbico social apresenta falhas na cognição, que tende a distorcer a avaliação de suas experiências interpessoais, desencadeando pensamentos patologicamente negativos acerca de si mesmo.

\section{Família como principal ponto de apoio}

A família tem fundamental importância para a formação do indivíduo, porque constitui a base, o alicerce principal para o desenvolvimento humano. Ela é essencial em todas as etapas do tratamento e da reinserção social do indivíduo portador de transtorno mental. É com apoio da família que o paciente procura o psicólogo, o psiquiatra ou outro profissional de saúde para relatar os sintomas que apresenta. $\mathrm{O}$ adoecimento é um evento imprevisto que desorganiza o modo de funcionamento de uma família(17).

O desejo expressado por todos os integrantes da família é poder sentir paz interior, tentar ter uma vida normal e ser feliz com seus familiares, podendo trabalhar tranquilamente, controlar seus medos e parar de sofrer ${ }^{(18)}$.

[...] Lá em casa todos, incluindo minha esposa e meus fiIhos, se preocupam comigo (E01).

[...] Tive muito apoio, principalmente de meus amigos e da minha mãe. Não tenho que reclamar, só agradecer (E16).

[...] minha mulher sempre me deu muito apoio, cuida de mim com muito amor e carinho (E18).

É possível perceber, nos relatos dos pacientes, que o tratamento conta com a participação e o acompanhamento dos familiares. Logo, a recuperação será mais rápida e estável, pois estes estarão amparados e sentirão o carinho e a compreensão daqueles que os rodeiam.

Sem o apoio da família, o tratamento será prejudicado, dificultando o sucesso da recuperação, ou seja, o déficit de orientação às famílias pode fazer com que eclodam conflitos e descompassos entre o paciente e a dinâmica familiar, com prejuízos para o processo terapêutico.

Um recurso utilizado por profissionais de saúde do CAPS I para melhorar o convívio entre paciente e família é a terapia familiar e a terapia de grupo, em que todos vivenciam dificuldades encontradas diariamente e também são trabalhados os potenciais individuais de enfrentamento. O grupo fortalece os vínculos afetivos e auxilia todos os componentes a enfrentarem o tratamento para conviverem de forma saudável ${ }^{(6)}$.

\section{Mudanças na qualidade de vida dos usuários do CAPS}

O CAPS possui uma estrutura que oferece atividades diárias, planejadas para o entretenimento e a recuperação dos pacientes. Dentre elas, destacamos a terapia ocupacional, terapia em grupo, oficinas lúdicas, passeios realizados em diversos locais da cidade, salão de beleza e a realização de festas em diferentes períodos do ano. Todas estas atividades requerem a participação de familiares e $\operatorname{amigos}^{(19)}$. 
[...] Eu gosto muito desse lugar, é minha segunda casa, sem ele acho que não conseguiria seguir em frente. (E12).

[...] Quando eu venho para o CAPS, eu me sinto melhor, porque quando eu tenho raiva dá vontade de pegar, bater, espancar, e por aqui eu fico mais calmo. (E15).

[...] Eu vivo querendo vim pra cá, porque eu não quero sair de casa. Aqui eu encontro força, alegria, os amigos que a gente tem, eu saio daqui outra pessoa. (E20).

A proposta do CAPS é manter o indivíduo saudável e inseri-lo na sociedade. O paciente que frequenta o CAPS tem a oportunidade de lidar com outras pessoas que vivenciam o mesmo sofrimento psíquico e poderá trocar experiências parecidas, podendo interagir melhor dentro da sociedade em que vive. Dessa maneira, essa inserção que os pacientes apresentam irá ajudar na recuperação, fazendo com que haja uma melhor qualidade de vida.

De acordo com Ayres ${ }^{(20)}$, os profissionais de saúde devem utilizar todos os recursos que favorecem a hermenêutica para estimular e otimizar a emergência de novos discursos e novas tecnologias com vistas à humanização.

Nos CAPS, observa-se que as práticas de reabilitação funcionam a partir de ações que têm como finalidade fazer meIhorar e proteger os portadores de sofrimento psíquico, mas que também indicam e impõem a direção em que se deseja obter uma transformação daquele que sofre e de sua relação com o mundo(19).

Da mesma forma, os pacientes acompanhados nos CAPS têm a oportunidade de lidar com outras pessoas portadoras da mesma patologia, ou de outras diferentes, mas serão capazes de trocar experiências parecidas, podendo interagir melhor dentro da sociedade em que vive.

O desafio aos serviços e profissionais de saúde é atender integralmente pessoas/famílias, criando espaços para uma atenção à saúde mental mais humanizada.

\section{CONSIDERAÇÕES FINAIS}

Este estudo permitiu-nos observar que os atuais Centros de Atenção Psicossociais estão desempenhando o seu papel, tendo em vista sua atitude de acolher o indivíduo com transtornos mentais, a partir de um acompanhamento interdisciplinar que proporciona a construção da cidadania e autonomia do sujeito e estimula sua integração social e familiar.

Os usuários investigados que se encontravam com diagnóstico de transtorno fóbico-ansioso caracterizam-se em sua maioria pelo gênero feminino, entre a faixa etária de 30 e 55 anos, de baixo nível de escolaridade. Sendo possível inferir que tais indicadores podem influenciar de forma significativa no desenvolvimento de sofrimento psíquico e, consequentemente, no transtorno pesquisado.

No que se refere às formas de enfrentamento utilizadas pelos usuários para melhorar os sintomas desse transtorno, destaca-se, nos discursos dos entrevistados que a família desempenha um papel fundamental no processo de adesão, tratamento e readaptação do portador de transtorno fóbico-ansioso. Além disso, a atuação da equipe de profissionais mostrou-se eficaz e capacitada para oferecer apoio psicoemocional e institucional.

Identifica-se que as pessoas portadoras de transtorno mental ainda relatam histórias de preconceitos e exclusão social sendo consideradas, por elas, muitas vezes como uma ameaça e perigo para sociedade. No entanto, os profissionais que atuam no CAPS aparecem como um mediador para socializar novamente o indivíduo, dentro de sua cultura, lazer e religião.

Dessa forma, o campo da saúde mental evolui cada vez mais, pois, diante do estudo, foi possível observar nos relatos uma gratidão pelo apoio e a ajuda que essas pessoas recebem dos profissionais e da instituição. Suscitando, também sua autoestima, o fortalecimento de vínculos além de se constatar que esse tratamento e atenção ajudam, ajudam no enfrentamento dos sintomas biopsicossociais. A prática do cuidado humanizado garante e estimula a integração entre os processos de trabalho e os projetos de vida dos usuários.

Percebeu-se, portanto, que responder à complexidade de cuidar de usuários com transtornos fóbico-ansiosos colocados à margem do convívio social configura-se em um desafio. Logo, os profissionais do serviço pesquisado contribuem para a construção de uma política pública de saúde mental mais humanizada, ficando clara a necessidade de um permanente esforço dos gestores públicos na construção de uma política de saúde de promoção da saúde e de bem-estar social.

\section{REFERÊNCIAS}

1. Organización Mundial de la Salud. Informe sobre la salud en el mundo 2003. Forjemos el futuro. Ginebra: Organización Mundial de la Salud; 2003.

2. Ministério da Saúde. Saúde mental no SUS: os centros de atenção psicossocial. Brasília: Ministério da Saúde; 2004.

3. Pelisoli CL, Moreira AK. Caracterização epidemiológica dos usuários do Centro de Atenção Psicossocial Casa Aberta. Rev Psiquiatr Rio Gd Sul 2005;27:270-7.

4. Rocha RM, Bartmann M, Kritz S. Enfermagem em saúde mental. 2. ed. Rio de Janeiro: SENAC; 1996.
5. Monteiro CB. O enfermeiro nos novos dispositivos assistenciais em saúde mental. Esc Anna Nery Enferm [periódico na internet].2006 [acesso 29 jul 2011];10(4):735-739. Disponível em: http://www.scielo.br/scielo.php?pid=S1414$-81452006000400017 \&$ script $=$ sci_arttext

6. Sadock BJ, Sadock VA. Compêndio de psiquiatria. 9. ed. Porto Alegre: Artmed; 2007.

7. Castillo ARGL, Recondo R, Asbahr FR, Manfro GG. Transtornos de ansiedade. Rev Bras Psiquiatr 2000:22(Suppl.2): 20-23. 
8. Palomba GA. Tratado de Psiquiatria Forense, Civil e Penal. São Paulo: Atheneu ; 2003.

9. Albano AM, Detweiler MF. The developmental and clinical impact of social anxiety and social phobia in children and adolescents. In: Hoffmann SG, Dibartolo PM, editores. From social anxiety to social phobia: multiple perspectives. Boston: Allyn \& Bacon; 2001. p. 162-78.

10. Prebianchi HB, Falleiros GG. Doença mental: representações de usuários e de profissionais da saúde. Psicol Estud 2011;16(1):33-41.

11. Bardin L. Análise de conteúdo. Lisboa: Edições 70; 2009.

12. Minayo MCS. O desafio do conhecimento: pesquisa qualitativa em saúde. 3. ed. São Paulo: Editora Hucitec; 1994.

13. Zerbetto SE, Efigênio EB, Santos NLN, Martins SC. O trabalho em um Centro de Atenção Psicossocial: dificuldades e facilidades da equipe de enfermagem. Rev Eletrônica Enferm 2011;13(1):99-109.

14. Mineka S, Ohman A. Born to fear: non-associative vs associative factors in the etiology of phobias. Behav Res Ther 2002;40(2):173-184.

15. Nardi AE. O tratamento farmacológico da fobia social. Rev Bras Psiquiatr [periódico na internet]. 1999 Dez [acesso em 29 jul 2011];21(4):249-257. Disponível em: http://www.scielo.br/scielo.php?pid = S1516-444619990 00400015\&script $=$ sci arttext

16. D’El Rey GJF, Pacini CA. Terapia cognitivo-comportamental da fobia social: modelos e técnicas. Psicol Estud 2006;11(2):269-275.

17. Schrank G, Olschowsky A. O centro de Atenção Psicossocial e as estratégias para inserção da família. Rev Esc Enferm USP 2008;42(1):127-34.

18. Nunes $M$, Torrenté $M$, Ottoni $V$, Moraes Neto $V$, Santana M. A dinâmica do cuidado em saúde mental: signos, significados e práticas de profissionais em um Centro de Assistência Psicossocial em Salvador, Bahia, Brasil. Cad Saúde Pública [periódico na internet]. 2008 Jan [acesso em 29 jul 2011];24(1):188-196. Disponível em: http://www. scielo.br/scielo.php?script $=$ sci arttext\&pid $=$ S0102$-311 \times 2008000100019$

19. Ballarin MLGS, Miranda IMS, Fuentes ACRC. Centro de atenção psicossocial: panorama das publicações de 1997 a 2008. Psicol Ciênc Prof [periódico na internet]. 2010 [acesso 29 jul 2011]:30(4):726-737. Disponível em: http://www.scielo.br/scielo.php?script=sci_arttext\&pid $=$ S1414-98932010000400005

20. Ayres JRCM. Hermenêutica e humanização das práticas de saúde. Cienc Saúde Coletiva 2005;10(3):549-60. 